Supplemental digital content for Cook DA, Brydges R, Zendejas B, Hamstra SJ, Hatala R. Mastery learning for health professionals using technology-enhanced simulation: A systematic review and meta-analysis. Acad Med. 2013;88(8).

\title{
Supplemental Digital Table 1
}

Key Features of Studies of Mastery Learning for Health Professionals Using Technology-Enhanced Simulation *

\begin{tabular}{|c|c|c|c|c|c|c|c|c|c|c|c|}
\hline $\begin{array}{l}\text { Author (year), } \\
\text { citation }\end{array}$ & Topic & $\begin{array}{l}\text { Participant } \\
\text { N; training * }\end{array}$ & $\begin{array}{l}\text { Study } \\
\text { design }\end{array}$ & $\begin{array}{l}\text { Comparison } \\
\text { cluster }^{\ddagger}\end{array}$ & $\begin{array}{l}\text { Instructional } \\
\text { features } \\
\S\end{array}$ & $\begin{array}{l}\text { Outcomes } \\
\text { reported } \pi\end{array}$ & Obj.' & Blind** & $\begin{array}{l}\text { Follow- } \\
\text { up }^{\text {t† }}\end{array}$ & MERSQI ${ }^{\ddagger \ddagger}$ & $\operatorname{NoS}^{\S \S}$ \\
\hline $\begin{array}{l}\text { Salvendy G } \\
(1980)^{23}\end{array}$ & Open surgery/suturing & 36; MS & $\mathrm{RCT}$ & $\begin{array}{r}\text { OE } \\
\text { SS: SM, FB }\end{array}$ & & ST, SPc & 1 & 1 & 1 & 13.5 & 4 \\
\hline $\begin{array}{l}\text { Stewart RD } \\
(1984)^{24}\end{array}$ & Intubation & 122; EMT & $2 \mathrm{NR}$ & SS: MM & $\mathrm{Cl}$ & $P$ & 1 & 0 & 1 & 12 & 4 \\
\hline Feil PH (1989) ${ }^{55}$ & Dentistry & $78 ; \mathrm{D}$ & $2 N R$ & $\mathrm{NI}$ & $\mathrm{Cl}, \mathrm{FB}$, Time & SPd & 1 & 1 & 0 & 12 & 3 \\
\hline $\begin{array}{l}\text { Stratton SJ } \\
(1991)^{25}\end{array}$ & Intubation & 125; EMT & RCT & SS: MM & $\mathrm{Cl}, \mathrm{FB}, \mathrm{Reps}$ & $\mathrm{P}$ & 0 & 0 & 1 & 11 & 3 \\
\hline Ashurst N (1996) ${ }^{56}$ & Intubation & 49; MD, RN & 1PP & $\mathrm{NI}$ & & SPC & 1 & 0 & 0 & 11 & 0 \\
\hline $\begin{array}{l}\text { Limpaphayom K } \\
(1997)^{20}\end{array}$ & Obstetrics & $300 ; 0$ & $2 \mathrm{NR}$ & $\begin{array}{r}\text { OE } \\
\text { Cost }\end{array}$ & $\mathrm{Cl}$, Time & $\mathrm{R}, \mathrm{K}, \mathrm{BP}, \mathrm{P}$ & 1 & 1 & 1 & 14 & 4 \\
\hline Kovacs G (2000) ${ }^{32}$ & Intubation & 84; MS, D, O & RCT & SS: EP & $\begin{array}{r}\mathrm{Cl}, \mathrm{FB}, \mathrm{Reps}, \\
\text { Time }\end{array}$ & SPc & 1 & 1 & 1 & 14.5 & 5 \\
\hline Naik VN (2001) ${ }^{21}$ & Intubation & $24 ; P G$ & RCT & $\mathrm{OE}$ & $\mathrm{Cl}$ & $\mathrm{BT}, \mathrm{BP}, \mathrm{P}$ & 1 & 1 & 1 & 16 & 4 \\
\hline Ferlitsch A (2002) ${ }^{5 /}$ & Endoscopy & 13; MS & RCT & $\mathrm{NI}$ & $\mathrm{Cl}$, Time & ST, SPC & 1 & 1 & 0 & 12.5 & 3 \\
\hline $\begin{array}{l}\text { Hamilton EC } \\
(2002)^{41}\end{array}$ & Min. invasive surg. & 50; PG & $\mathrm{RCT}$ & SS: SM & Cl, Reps, Time & $\begin{array}{r}\mathrm{R}, \mathrm{ST}, \mathrm{SPC} \\
\mathrm{BP}\end{array}$ & 1 & 1 & 1 & 14 & 5 \\
\hline $\begin{array}{l}\text { Seymour NE } \\
(2002)^{58}\end{array}$ & Min. invasive surg. & 16; PG & RCT & $\mathrm{NI}$ & $\mathrm{Cl}$, FB, Time & $\mathrm{BT}, \mathrm{BP}$ & 1 & 1 & 0 & 14 & 3 \\
\hline $\begin{array}{l}\text { Neumann M } \\
(2003)^{59}\end{array}$ & Endoscopy & $\begin{array}{r}25 ; \mathrm{MS}, \mathrm{PG}, \\
\mathrm{MD}\end{array}$ & 1PP & $\mathrm{NI}$ & $\mathrm{Cl}, \mathrm{FB}$, Time & SPc & 1 & 0 & 0 & 12 & 0 \\
\hline $\begin{array}{l}\text { Jacomides L } \\
(2004)^{60}\end{array}$ & Endoscopy & 32; MS, PG & $2 N R$ & $\mathrm{NI}$ & $\mathrm{Cl}$, Time & ST & 1 & 1 & 1 & 12.5 & 2 \\
\hline Mayo PH (2004) & $\begin{array}{l}\text { Anesthesia, Resuscitation (BLS, ACLS, } \\
\text { ATLS) }\end{array}$ & 50; PG & RCT & $\mathrm{NI}$ & $\mathrm{Cl}, \mathrm{FB}$ & SPc & 1 & 0 & 1 & 12.5 & 5 \\
\hline Ahlberg G (2005) & Endoscopy & 12; PG & RCT & $\mathrm{NI}$ & $\mathrm{Cl}, \mathrm{FB}$, Time & $\mathrm{BT}, \mathrm{P}$ & 1 & 1 & 0 & 15 & 4 \\
\hline $\begin{array}{l}\text { Korndorffer JR, Jr. } \\
(2005)^{63}\end{array}$ & Min. invasive surg. & 20; MS & RCT & $\mathrm{NI}$ & $\mathrm{Cl}$, Reps & ST, SPc & 1 & 1 & 1 & 13.5 & 5 \\
\hline $\begin{array}{l}\text { Korndorffer JR, Jr. } \\
(2005)^{64}\end{array}$ & Min. invasive surg. & 17; PG & RCT & $\mathrm{NI}$ & $\mathrm{Cl}, \mathrm{FB}, \mathrm{Reps}$ & ST, SPc & 1 & 1 & 0 & 12.5 & 4 \\
\hline $\begin{array}{l}\text { Stefanidis D } \\
(2005)^{65}\end{array}$ & Min. invasive surg. & $14 ; P G$ & 1PP & $\mathrm{NI}$ & & SPC & 1 & 1 & 1 & 12 & 2 \\
\hline Tanoue K (2005) ${ }^{22}$ & Min. invasive surg. & 35; MS & RCT & $\mathrm{OE}$ & $\mathrm{Cl}$ & ST, SPc & 1 & 1 & 1 & 12.5 & 4 \\
\hline Uchal M (2005) & Min. invasive surg. & $17 ; \mathrm{PG}$ & RCT & SS: Other & Time & $\mathrm{SPC}$ & 1 & 1 & 1 & 15.5 & 6 \\
\hline $\begin{array}{l}\text { Andreatta PB } \\
(2006)^{66}\end{array}$ & Min. invasive surg. & 21; PG & RCT & $\mathrm{NI}$ & $\mathrm{Cl}$, Reps & ST, SPC & 1 & 1 & 1 & 13.5 & 5 \\
\hline
\end{tabular}

Copyright $\odot$ by the Association of American Medical Colleges. Unauthorized reproduction is prohibited. 
Supplemental digital content for Cook DA, Brydges R, Zendejas B, Hamstra SJ, Hatala R. Mastery learning for health professionals using technology-enhanced simulation: A systematic review and meta-analysis. Acad Med. 2013;88(8).

\begin{tabular}{|c|c|c|c|c|c|c|c|c|c|c|c|}
\hline $\begin{array}{l}\text { Author (year), } \\
\text { citation }\end{array}$ & Topic & $\begin{array}{l}\text { Participant } \\
\text { N; training * }\end{array}$ & $\begin{array}{l}\text { Study } \\
\text { design }^{\dagger}\end{array}$ & $\begin{array}{l}\text { Comparison } \\
\text { cluster }{ }^{\ddagger}\end{array}$ & $\begin{array}{l}\text { Instructional } \\
\text { features } \S\end{array}$ & $\begin{array}{l}\text { Outcomes } \\
\text { reported } \pi\end{array}$ & Obj.' & Blind** & $\begin{array}{l}\text { Follow- } \\
\text { up }^{\dagger+}\end{array}$ & MERSQI & $\operatorname{NOS}^{\S \S}$ \\
\hline $\begin{array}{l}\text { Rosenthal ME } \\
(2006)^{45}\end{array}$ & Resuscitation (BLS, ACLS, ATLS) & $49 ; P G$ & $\mathrm{RCT}$ & SS: Other & $\mathrm{Cl}, \mathrm{FB}$ & $\mathrm{SPC}$ & 1 & 0 & J 1 & 12.5 & 4 \\
\hline $\begin{array}{l}\text { Scerbo MW } \\
(2006)^{43}\end{array}$ & Venous access & 20; MS & RCT & SS: SM & FB & SPc & 1 & 0 & 1 & 12.5 & 3 \\
\hline $\begin{array}{l}\text { Stefanidis D } \\
(2006)^{67}\end{array}$ & Min. invasive surg. & 18; PG, MD & $1 P P$ & $\mathrm{NI}$ & $\mathrm{Cl}, \mathrm{Reps}$ & ST, SPc, SPd & 1 & 0 & 1 & 12 & 1 \\
\hline $\begin{array}{l}\text { Stefanidis D } \\
(2006)^{33}\end{array}$ & Min. invasive surg. & 18; MS & RCT & SS: EP & $\begin{array}{r}\mathrm{Cl}, \mathrm{FB}, \begin{array}{r}\text { Reps, } \\
\text { Time }\end{array}\end{array}$ & SPc & 1 & 0 & 0 & 11.5 & 3 \\
\hline $\begin{array}{l}\text { Stefanidis D } \\
(2006)^{68}\end{array}$ & Min. invasive surg. & 20; PG & $1 \mathrm{PP}$ & $\mathrm{NI}$ & $\mathrm{Cl}$, Reps, Time & ST, SPc & 1 & 1 & 1 & 11 & 2 \\
\hline Wayne DB (2006) ${ }^{69}$ & Resuscitation (BLS, ACLS, ATLS) & $41 ; P G$ & 1PP & $\mathrm{NI}$ & $\mathrm{Cl}, \mathrm{FB}$, Time & SPC & 1 & 0 & b & 13 & 2 \\
\hline $\begin{array}{l}\text { Aggarwal R } \\
(2007)^{70}\end{array}$ & Min. invasive surg. & 20; PG & RCT & $\mathrm{NI}$ & $\mathrm{Cl}$, Reps & ST, SPC & 1 & 1 & 1 & 15.5 & 5 \\
\hline Ahlberg G (2007) & Min. invasive surg. & 13; PG & RCT & $\mathrm{NI}$ & $\begin{array}{r}\mathrm{Cl}, \mathrm{FB}, \text { Reps, } \\
\text { Time }\end{array}$ & $\mathrm{BT}, \mathrm{BP}, \mathrm{P}$ & 1 & 1 & 1 & 17 & 5 \\
\hline $\begin{array}{l}\text { Cosman } \mathrm{PH} \\
(2007)^{72}\end{array}$ & Min. invasive surg. & 10; PG & RCT & $\mathrm{NI}$ & & BT, BP & 1 & 1 & 0 & 14 & 3 \\
\hline Ganai S (2007) & Min. invasive surg. & 20; MS & RCT & $\mathrm{NI}$ & $\mathrm{Cl}, \mathrm{Reps}$ & ST, SPC & 1 & 0 & D & 12.5 & 4 \\
\hline Kory PD (2007) & Resuscitation (BLS, ACLS, ATLS) & $62 ; P G$ & $2 N R$ & $\mathrm{NI}$ & $\mathrm{Cl}, \mathrm{FB}$ & $\mathrm{SPC}$ & 1 & 0 & 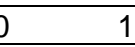 & 11.5 & 2 \\
\hline Scott DJ (2007) & Open surgery/suturing & $4 ; P G$ & 1PP & $\mathrm{NI}$ & Reps, Time & SPc & 0 & 0 & 1 & 10 & 1 \\
\hline $\begin{array}{l}\text { Stefanidis D } \\
(2007)^{46}\end{array}$ & Min. invasive surg. & $32 ; \mathrm{MS}$ & RCT & $\begin{array}{r}\mathrm{NI} \\
\text { SS: Other }\end{array}$ & $\mathrm{Cl}$, Reps, Time & SPc & 1 & 0 & 1 & 12.5 & 4 \\
\hline $\begin{array}{l}\text { Stefanidis D } \\
(2007)^{39}\end{array}$ & Min. invasive surg. & $34 ; \mathrm{MS}$ & $2 \mathrm{NR}$ & SS: FB & $\mathrm{Cl}, \mathrm{FB}, \mathrm{Reps}$ & SPc & 1 & 0 & 1 & 11.5 & 1 \\
\hline Yi SY $(2007)^{16}$ & Endoscopy & 9;PG & 1PP & $\mathrm{NI}$ & Reps & ST, SPC & 1 & 1 & 0 & 10 & 1 \\
\hline Goova MT (2008) $)^{\prime \prime}$ & Open surgery/suturing & $37 ; P G$ & 1PP & $\mathrm{NI}$ & Cl, Reps, Time & SPc & 1 & 0 & 1 & 13 & 2 \\
\hline $\begin{array}{l}\text { Lammers RL } \\
(2008)^{29}\end{array}$ & Epistaxis management & 28; PG & RCT & SS: EP & $\mathrm{Cl}, \mathrm{FB}$ & ST, SPc & 1 & 1 & 1 & 14.5 & 5 \\
\hline Scott DJ (2008) & Min. invasive surg. & $21 ; M S$ & 1PP & $\mathrm{NI}$ & $\begin{array}{r}\mathrm{Cl}, \mathrm{FB}, \text { Reps, } \\
\text { Time }\end{array}$ & SPc & 1 & 0 & 1 & 12.5 & 1 \\
\hline Smith KK (2008) ${ }^{79}$ & Resuscitation (BLS, ACLS, ATLS) & 104; RN & 1PP & $\mathrm{NI}$ & $\mathrm{Cl}$ & SPc & 1 & 0 & 0 & 11 & 0 \\
\hline $\begin{array}{l}\text { Stefanidis D } \\
(2008)^{80}\end{array}$ & Min. invasive surg. & 15; MS & RCT & $\mathrm{NI}$ & $\begin{array}{r}\mathrm{Cl}, \mathrm{FB}, \text { Reps, } \\
\text { Time }\end{array}$ & SPc & 1 & 0 & 1 & 14.5 & 3 \\
\hline $\begin{array}{l}\text { Van Sickle KR } \\
(2008)^{81}\end{array}$ & Min. invasive surg. & $24 ; P G$ & RCT & $\mathrm{NI}$ & $\mathrm{Cl}, \mathrm{FB}$ & BT, BP & 1 & 1 & 1 & 15 & 4 \\
\hline Wayne DB (2008) ${ }^{82}$ & Percutaneous proc. & 40; PG & 1PP & $\mathrm{NI}$ & $\mathrm{Cl}, \mathrm{FB}$ & $\mathrm{K}, \mathrm{SPC}$ & 1 & 1 & 1 & 13 & 3 \\
\hline Yi SY $(2008)^{83}$ & Endoscopy & $11 ; P G$ & $2 N R$ & $\mathrm{NI}$ & $\mathrm{Cl}$, Reps, Time & $\mathrm{BT}, \mathrm{BP}, \mathrm{P}$ & 0 & 0 & 1 & 10 & 2 \\
\hline Barsuk JH (2009) $^{84}$ & Venous access & 18; PG & $2 \mathrm{NR}$ & $\mathrm{NI}$ & $\mathrm{Cl}, \mathrm{FB}$ & SPC & 1 & 0 & 1 & 15.5 & 3 \\
\hline Barsuk JH (2009) ${ }^{85}$ & Venous access & 103; PG & $2 \mathrm{NR}$ & $\mathrm{NI}$ & $\mathrm{Cl}$ & $\mathrm{BP}, \mathrm{P}$ & 0 & 0 & 1 & 10 & 3 \\
\hline${\text { Barsuk JH }(2009)^{86}}^{86}$ & Venous access & 92; PG & $2 \mathrm{NR}$ & $\mathrm{NI}$ & $\mathrm{Cl}, \mathrm{FB}$ & $\mathrm{P}$ & 1 & 0 & 0 & 13 & 2 \\
\hline Barsuk JH (2009) & Venous access & 41; PG & $2 \mathrm{NR}$ & $\mathrm{NI}$ & $\mathrm{Cl}, \mathrm{FB}$ & $\mathrm{SPC}, \mathrm{BP}, \mathrm{P}$ & 1 & 1 & 1 & 15 & 4 \\
\hline Britt RC $(2009)^{88}$ & Venous access & $34 ; P G$ & RCT & $\mathrm{NI}$ & & $\mathrm{BP}, \mathrm{P}$ & 1 & 0 & 0 & 13 & 3 \\
\hline
\end{tabular}

Copyright $\odot$ by the Association of American Medical Colleges. Unauthorized reproduction is prohibited. 
Supplemental digital content for Cook DA, Brydges R, Zendejas B, Hamstra SJ, Hatala R. Mastery learning for health professionals using technology-enhanced simulation: A systematic review and meta-analysis. Acad Med. 2013;88(8).

\begin{tabular}{|c|c|c|c|c|c|c|c|c|c|c|c|}
\hline $\begin{array}{l}\text { Author (year), } \\
\text { citation }\end{array}$ & Topic & $\begin{array}{l}\text { Participant } \\
\text { N; training * }\end{array}$ & $\begin{array}{l}\text { Study } \\
\text { design }\end{array}$ & $\begin{array}{l}\text { Comparison } \\
\text { cluster }^{\ddagger}\end{array}$ & $\begin{array}{l}\text { Instructional } \\
\text { features } \\
\S\end{array}$ & $\begin{array}{l}\text { Outcomes } \\
\text { reported } \pi\end{array}$ & Obj.' & Blind** & $\begin{array}{l}\text { Follow- } \\
\text { up }\end{array}$ & MERSQI ${ }^{\ddagger \ddagger}$ & $\operatorname{NOS}^{\S \S}$ \\
\hline $\begin{array}{l}\text { Castellvi AO } \\
(2009)^{89}\end{array}$ & Min. invasive surg. & $44 ; P G$ & $1 \mathrm{PP}$ & $\mathrm{NI}$ & & $\mathrm{SPc}$ & 1 & 0 & 0 & $1 \quad 12$ & 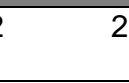 \\
\hline $\begin{array}{l}\text { Domuracki KJ } \\
(2009)^{26}\end{array}$ & Intubation & 101; MS, RN & RCT & SS: MM, FB & & SPc & 1 & 1 & 1 & 13.5 & 5 \\
\hline $\begin{array}{l}\text { Duncan DR } \\
(2009)^{90}\end{array}$ & Percutaneous proc. & 5; MD & 1PP & $\mathrm{NI}$ & $\mathrm{Cl}, \mathrm{FB}$ & $\mathrm{P}$ & 1 & 0 & 0 & 12.5 & 0 \\
\hline Essani R (2009) $^{91}$ & Min. invasive surg. & 8; PG & 1PP & $\mathrm{NI}$ & $\mathrm{Cl}, \mathrm{FB}$ & ST, SPc & 1 & 1 & 1 & 14 & 2 \\
\hline $\begin{array}{l}\text { Feudner EM } \\
(2009)^{92}\end{array}$ & Microsurgery/Ophthalmology & 63; MS, PG & $2 N R$ & $\mathrm{NI}$ & $\mathrm{Cl}$, Reps & $\mathrm{SPC}$ & 1 & 1 & 1 & 13.5 & 3 \\
\hline $\begin{array}{l}\text { Hoadley TA } \\
(2009)^{42}\end{array}$ & Resuscitation (BLS, ACLS, ATLS) & $\begin{array}{r}\text { 53; MD, RN, } \\
\text { EMT, O }\end{array}$ & RCT & SS: SM & $\mathrm{Cl}$, FB, Time & $\mathrm{R}, \mathrm{K}, \mathrm{SPC}$ & 1 & 0 & 0 & 14 & 2 \\
\hline Hogle NJ (2009) ${ }^{93}$ & Min. invasive surg. & $13 ; \mathrm{PG}$ & RCT & $\mathrm{NI}$ & & $\mathrm{BP}$ & 1 & 1 & 1 & 15 & 4 \\
\hline Larsen CR (2009) $^{94}$ & Min. invasive surg. & $24 ; P G$ & RCT & $\mathrm{NI}$ & Time & $\mathrm{BT}, \mathrm{BP}$ & 1 & 1 & 1 & 17 & 6 \\
\hline Lubin J (2009) $^{95}$ & Intubation & 17; RN, EMT & $1 \mathrm{PP}$ & $\mathrm{NI}$ & Reps, Time & $\mathrm{P}$ & 1 & 0 & 0 & 12.5 & 0 \\
\hline $\begin{array}{l}\text { McDougall EM } \\
(2009)^{35}\end{array}$ & Min. invasive surg. & $20 ; M S$ & RCT & $\begin{array}{r}\text { SS: SM } \\
\text { Cost }\end{array}$ & $\mathrm{Cl}, \mathrm{FB}$ & $\mathrm{R}, \mathrm{ST}, \mathrm{SPC}$ & 1 & 1 & 1 & 15.5 & 4 \\
\hline Naylor RA (2009) ${ }^{96}$ & $\begin{array}{l}\text { Open surgery/suturing, Natural orifice } \\
\text { proc., Physical exam }\end{array}$ & 204; MS & 1PP & $\mathrm{NI}$ & $\mathrm{Cl}$ & SPc & 1 & 0 & 0 & 12 & 2 \\
\hline $\begin{array}{l}\text { Pauly-O'Neill S } \\
(2009)^{97}\end{array}$ & med administration & 39; RN & 1PP & $\mathrm{NI}$ & & $\mathrm{SPC}$ & 1 & 0 & 0 & 8 & 0 \\
\hline $\begin{array}{l}\text { Rosenthal ME } \\
(2009)^{30}\end{array}$ & Min. invasive surg. & 20; MS & $2 \mathrm{NR}$ & $\begin{array}{r}\text { SS: EP } \\
\text { Cost }\end{array}$ & Reps, Time & ST, SPc & 1 & 0 & 0 & 11.5 & 1 \\
\hline $\begin{array}{l}\text { Stefanidis D } \\
(2009)^{98}\end{array}$ & Min. invasive surg. & 11; MS, PG & 1PP & $\mathrm{NI}$ & Reps & ST, SPc & 1 & 1 & 1 & 13 & 2 \\
\hline Yi SY $(2009)^{99}$ & Endoscopy & 9; PG & 1PP & $\mathrm{NI}$ & $\mathrm{Cl}$, Reps & ST, SPc & 1 & 1 & 1 & 10 & 1 \\
\hline Brydges R (2010) & Venous access & $60 ; \mathrm{RN}$ & RCT & SS: MM, SR & Reps & $\mathrm{SPC}$ & 1 & 1 & 1 & 14.5 & 5 \\
\hline Butter J (2010) ${ }^{100}$ & Physical exam & 108; MS & $2 \mathrm{NR}$ & $\mathrm{NI}$ & $\mathrm{Cl}$, Reps & $\mathrm{K}, \mathrm{SPC}$ & 1 & 1 & 1 & 13.5 & 3 \\
\hline Evans LV (2010) $)^{101}$ & Venous access & 188; PG & RCT & $\mathrm{NI}$ & $\mathrm{Cl}, \mathrm{FB}, \mathrm{Reps}$ & $\mathrm{SPC}, \mathrm{BP}, \mathrm{P}$ & 1 & 1 & 1 & 15 & 5 \\
\hline Fried MP $(2010)^{102}$ & Min. invasive surg. & 28; PG & RCT & $\mathrm{NI}$ & & $\mathrm{BT}, \mathrm{BP}$ & 1 & 1 & 1 & 16 & 5 \\
\hline $\begin{array}{l}\text { Gauger PG } \\
(2010)^{27}\end{array}$ & Min. invasive surg. & $14 ; P G$ & RCT & SS: MM, SR & Reps, Time & $\mathrm{SPC}, \mathrm{BP}$ & 1 & 1 & 1 & 13 & 3 \\
\hline $\begin{array}{l}\text { Kallstrom R } \\
(2010)^{103}\end{array}$ & Endoscopy & $24 ; P G$ & 1PP & $\mathrm{NI}$ & $\mathrm{Cl}$, Reps, Time & $\mathrm{BT}, \mathrm{BP}, \mathrm{P}$ & 1 & 1 & 1 & 13 & 1 \\
\hline $\begin{array}{l}\text { Molinas CR } \\
(2010)^{48}\end{array}$ & Min. invasive surg. & 60; PG, MD & RCT & $\begin{array}{r}\mathrm{NI} \\
\text { SS: Other }\end{array}$ & Reps & SPc & 1 & 1 & 1 & 11.5 & 3 \\
\hline $\begin{array}{l}\text { Rosenthal ME } \\
(2010)^{104}\end{array}$ & Min. invasive surg. & $21 ; M S$ & 1PP & $\mathrm{NI}$ & Reps & $\mathrm{SPC}$ & 1 & 0 & 0 & 11 & 0 \\
\hline Sroka G (2010) ${ }^{105}$ & Min. invasive surg. & $17 ; P G$ & RCT & $\mathrm{NI}$ & $\begin{array}{r}\mathrm{Cl}, \mathrm{FB}, \begin{array}{r}\text { Reps, } \\
\text { Time }\end{array}\end{array}$ & $\mathrm{SPC}, \mathrm{BP}$ & 1 & 1 & 1 & 16 & 5 \\
\hline $\begin{array}{l}\text { Stefanidis D } \\
(2010)^{31}\end{array}$ & Min. invasive surg. & 20; MS & RCT & $\begin{array}{r}\text { SS: EP } \\
\text { Cost }\end{array}$ & $\begin{array}{r}\mathrm{Cl}, \mathrm{FB}, \mathrm{Reps}, \\
\text { Time }\end{array}$ & ST, SPc & 1 & 1 & 1 & 14.5 & 5 \\
\hline
\end{tabular}

Copyright $\odot$ by the Association of American Medical Colleges. Unauthorized reproduction is prohibited. 
Supplemental digital content for Cook DA, Brydges R, Zendejas B, Hamstra SJ, Hatala R. Mastery learning for health professionals using technology-enhanced simulation: A systematic review and meta-analysis. Acad Med. 2013;88(8).

\begin{tabular}{|c|c|c|c|c|c|c|c|c|c|c|}
\hline $\begin{array}{l}\text { Author (year), } \\
\text { citation }\end{array}$ & Topic & $\begin{array}{l}\text { Participant } \\
\text { N; training * }\end{array}$ & $\begin{array}{l}\text { Study } \\
\text { design }\end{array}$ & $\begin{array}{l}\text { Instructional } \\
\text { features }^{\S}\end{array}$ & $\begin{array}{l}\text { Outcomes } \\
\text { reported }\end{array}$ & Obj.' & Blind** & $\begin{array}{l}\text { Follow- } \\
\text { up }^{\text {t† }}\end{array}$ & MERSQI ${ }^{\ddagger \ddagger}$ & $\operatorname{NOS}^{\S \S}$ \\
\hline $\begin{array}{l}\text { Stefanidis D } \\
(2010)^{106}\end{array}$ & Min. invasive surg. & 15; PG & $1 \mathrm{PP}$ & $\mathrm{NI} \mathrm{Cl}$, Reps, Time & $\mathrm{SPc}$ & 0 & 0 & 0 & 6 & 0 \\
\hline $\begin{array}{l}\text { Unalan PC } \\
(2010)^{107}\end{array}$ & Min. invasive surg. & 64; PG, MD & $1 \mathrm{PP}$ & $\begin{array}{r}\mathrm{NI} \quad \mathrm{Cl}, \mathrm{FB}, \begin{array}{r}\text { Reps, } \\
\text { Time }\end{array} \\
\end{array}$ & SPc & 0 & 0 & 1 & 10 & 2 \\
\hline $\begin{array}{l}\text { Vankipuram M } \\
(2010)^{108}\end{array}$ & Open surgery/suturing & 10; MS & $2 \mathrm{NR}$ & $\mathrm{NI}$ & SPd & 1 & 1 & 0 & 10.5 & 3 \\
\hline Arora S (2011) ${ }^{34}$ & Min. invasive surg. & $18 ; P G$ & RCT & SS: EP & $\mathrm{SPc}$ & 1 & 1 & 1 & 14.5 & 5 \\
\hline $\begin{array}{l}\text { Maschuw K } \\
(2011)^{109}\end{array}$ & Min. invasive surg. & $50 ; P G$ & $\mathrm{RCT}$ & $\mathrm{NI} \mathrm{Cl}$, Reps, Time & $\mathrm{ST}, \mathrm{SPC}$ & 1 & 1 & 1 & 12.5 & 5 \\
\hline Panait L (2011) & Min. invasive surg. & 23; PG & 1PP & $\mathrm{NI}$ & SPc & 0 & 0 & 1 & 8 & 1 \\
\hline $\begin{array}{l}\text { Snyder CW } \\
(2011)^{37}\end{array}$ & Min. invasive surg., Endoscopy & 36; MS & $\mathrm{RCT}$ & $\mathrm{Cl}, \mathrm{FB}$, Time & ST & 1 & 1 & 1 & 13.5 & 4 \\
\hline $\begin{array}{l}\text { Thompson JR } \\
(2011)^{44}\end{array}$ & Min. invasive surg. & 33; MS, O & $\mathrm{RCT}$ & $\begin{array}{rl}\mathrm{NI} & \mathrm{Cl}, \mathrm{Reps}, \text { Time } \\
\text { SS: SM } & \end{array}$ & ST, SPc & 1 & 1 & 0 & 12 & 3 \\
\hline
\end{tabular}

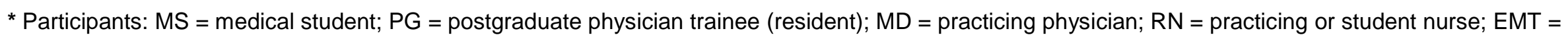
emergency medicine technician or other first responder; $\mathrm{D}=$ practicing or student dentist; $\mathrm{O}=$ other health professional.

${ }^{\dagger}$ Study design: 1PP = 1 group pre-post study; 2NR = nonrandomized 2-group study; RCT = randomized 2-group study.

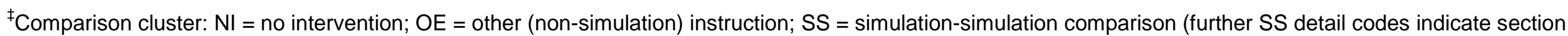

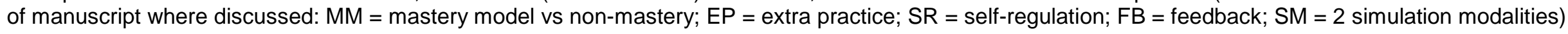

${ }^{\S}$ Instructional features: $\mathrm{Cl}=$ cognitive interactivity high; FB = feedback high; Reps = many repetitions; Time = learning time $\geq 5$ hours.

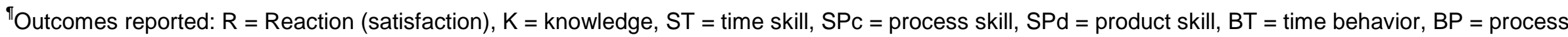
behavior, $\mathrm{P}=$ patient effects.

'Obj. = objective assessment (1) or self-report (0).

${ }^{* \star}$ Blind = blinded assessment (1) or unblinded (0).

${ }^{\dagger \dagger}$ Follow-up $=$ follow-up high $\geq 75 \%$ (1) or low/not reported $(0)$.

${ }^{\ddagger \ddagger}$ MERSQI = Medical Education Research Study Quality Instrument (maximum score 18).

${ }^{\S \S} \mathrm{NOS}=$ modified Newcastle-Ottawa scale (maximum score 6). 
Supplemental digital content for Cook DA, Brydges R, Zendejas B, Hamstra SJ, Hatala R. Mastery learning for health professionals using technology-enhanced simulation: A systematic review and meta-analysis. Acad Med. 2013;88(8).

\section{Supplemental Digital Table 2}

\section{Subgroup Analyses of Studies Comparing Simulation-Based Mastery Learning with No Intervention *}

Table 2.1. Subgroup Analyses for Outcome: Time Skill

\begin{tabular}{|c|c|c|c|c|c|}
\hline Feature & Subgroup & $\begin{array}{l}\text { No. studies (No. } \\
\text { participants) }\end{array}$ & ES & $\begin{array}{r}95 \% \text { confidence } \\
\text { interval }\end{array}$ & $\boldsymbol{P}_{\text {interaction }}$ \\
\hline All & & $15(278)$ & 0.94 & $0.64,1.24$ & \\
\hline \multirow[t]{3}{*}{ No. groups, timing } & 2-group posttest-only & $3(52)$ & 1.18 & $0.58,1.79$ & \\
\hline & 2-group pre-post & $6(151)$ & 0.76 & $0.3,1.22$ & \\
\hline & 1-group pre-post & $6(75)$ & 1.09 & $0.64,1.54$ & \\
\hline \multirow[t]{2}{*}{ Group allocation } & Randomized & $8(171)$ & 0.68 & $0.36,1.01$ & 0.004 \\
\hline & Nonrandomized & $1(32)$ & 1.62 & $1.07,2.17$ & \\
\hline \multirow[t]{4}{*}{ Participants } & Medical student & $6(109)$ & 1.2 & $0.44,1.95$ & \\
\hline & Postgraduate trainees & $11(212)$ & 1.03 & $0.71,1.35$ & \\
\hline & Practicing physicians & $1(18)$ & 1.37 & $0.65,2.08$ & \\
\hline & Other & $1(14)$ & 1.79 & $0.45,3.12$ & \\
\hline \multirow[t]{2}{*}{ Cognitive interactivity } & High & $13(258)$ & 0.9 & $0.6,1.2$ & 0.52 \\
\hline & Low & $2(20)$ & 1.59 & $-0.51,3.69$ & \\
\hline \multirow[t]{2}{*}{ Feedback } & High & $2(25)$ & 0.98 & $0.43,1.53$ & 0.9 \\
\hline & Low & $13(253)$ & 0.94 & $0.61,1.27$ & \\
\hline \multirow[t]{2}{*}{ Repetitions } & Many $>10$ & $12(225)$ & 0.81 & $0.51,1.1$ & 0.01 \\
\hline & Few $\leq 9$ & $3(53)$ & 1.56 & $1.09,2.03$ & \\
\hline \multirow[t]{2}{*}{ Time learning } & $\geq 5$ hours & $5(129)$ & 1.11 & $0.56,1.67$ & 0.64 \\
\hline & $<5$ hours & $9(130)$ & 0.96 & $0.65,1.27$ & \\
\hline \multirow[t]{2}{*}{ Assessment } & Blinded & $12(221)$ & 1.05 & $0.73,1.37$ & 0.28 \\
\hline & Not blinded & $3(57)$ & 0.61 & $-0.13,1.34$ & \\
\hline \multirow[t]{2}{*}{ NOS } & High $\geq 4$ & $6(144)$ & 0.56 & $0.26,0.86$ & 0.003 \\
\hline & Low $<4$ & $9(134)$ & 1.25 & $0.89,1.6$ & \\
\hline \multirow[t]{2}{*}{ MERSQI } & High $\geq 12$ & $12(240)$ & 1.03 & $0.65,1.4$ & 0.36 \\
\hline & Low $<12$ & $3(38)$ & 0.77 & $0.36,1.18$ & \\
\hline Effect size estimation & Precise & $13(245)$ & 0.94 & $0.6,1.28$ & \\
\hline
\end{tabular}

Copyright $\odot$ by the Association of American Medical Colleges. Unauthorized reproduction is prohibited. 
Supplemental digital content for Cook DA, Brydges R, Zendejas B, Hamstra SJ, Hatala R. Mastery learning for health professionals using technology-enhanced simulation: A systematic review and meta-analysis. Acad Med. 2013;88(8).

Table 2.2. Subgroup Analyses for Outcome: Process Skill

\begin{tabular}{|c|c|c|c|c|c|}
\hline Feature & Subgroup & $\begin{array}{r}\text { No. studies (No. } \\
\text { participants) }\end{array}$ & ES & $\begin{array}{r}95 \% \text { confidence } \\
\text { interval }\end{array}$ & $\boldsymbol{P}_{\text {interaction }}$ \\
\hline All & & $41(1523)$ & 1.29 & $1.08,1.5$ & \\
\hline \multirow[t]{3}{*}{ No. groups, timing } & 2-group posttest-only & $8(466)$ & 1.04 & $0.58,1.5$ & \\
\hline & 2-group pre-post & $11(320)$ & 1.16 & $0.8,1.53$ & \\
\hline & 1-group pre-post & $22(737)$ & 1.45 & $1.16,1.74$ & \\
\hline \multirow[t]{2}{*}{ Group allocation } & Randomized & $14(495)$ & 1.11 & $0.76,1.46$ & 0.99 \\
\hline & Nonrandomized & $5(291)$ & 1.11 & $0.68,1.54$ & \\
\hline \multirow[t]{5}{*}{ Participants } & Medical student & $13(510)$ & 1.45 & $1.06,1.83$ & \\
\hline & Postgraduate trainees & $28(959)$ & 1.27 & $1.01,1.53$ & \\
\hline & Practicing physicians & $5(165)$ & 1.1 & $0.6,1.59$ & \\
\hline & Nurses & $3(152)$ & 1.3 & $0.71,1.9$ & \\
\hline & Other & $2(78)$ & 0.7 & $0.03,1.36$ & \\
\hline \multirow[t]{2}{*}{ Cognitive interactivity } & High & $32(1340)$ & 1.27 & $1.03,1.5$ & 0.90 \\
\hline & Low & $8(153)$ & 1.3 & $0.83,1.77$ & \\
\hline \multirow[t]{2}{*}{ Feedback } & High & $14(603)$ & 1.41 & $0.98,1.84$ & 0.31 \\
\hline & Low & $25(875)$ & 1.16 & $0.93,1.38$ & \\
\hline \multirow[t]{2}{*}{ Repetitions } & Many >10 & $25(825)$ & 1.3 & $1,1.6$ & 0.87 \\
\hline & Few $\leq 9$ & $15(668)$ & 1.27 & $1.02,1.52$ & \\
\hline \multirow[t]{2}{*}{ Time learning } & $\geq 5$ hours & $14(353)$ & 1.49 & $1.04,1.94$ & 0.49 \\
\hline & $<5$ hours & $19(625)$ & 1.31 & $1.06,1.57$ & \\
\hline \multirow[t]{2}{*}{ Assessment } & Blinded & $16(486)$ & 1.04 & $0.74,1.34$ & 0.06 \\
\hline & Not blinded & 25 (1037) & 1.43 & $1.16,1.69$ & \\
\hline \multirow[t]{2}{*}{ NOS } & High $\geq 4$ & $11(454)$ & 0.97 & $0.6,1.33$ & 0.05 \\
\hline & Low $<4$ & 30 (1069) & 1.4 & $1.17,1.63$ & \\
\hline \multirow[t]{2}{*}{ MERSQI } & High $\geq 12$ & $28(1110)$ & 1.36 & $1.1,1.63$ & 0.32 \\
\hline & Low $<12$ & $13(413)$ & 1.14 & $0.81,1.48$ & \\
\hline Effect size estimation & Precise & $39(1490)$ & 1.3 & $1.09,1.52$ & \\
\hline
\end{tabular}

Copyright $\odot$ by the Association of American Medical Colleges. Unauthorized reproduction is prohibited. 
Supplemental digital content for Cook DA, Brydges R, Zendejas B, Hamstra SJ, Hatala R. Mastery learning for health professionals using technology-enhanced simulation: A systematic review and meta-analysis. Acad Med. 2013;88(8).

Table 2.3. Subgroup Analyses for Outcome: Time Behavior

\begin{tabular}{|c|c|c|c|c|c|}
\hline Feature & Subgroup & $\begin{array}{l}\text { No. studies (No. } \\
\text { participants) }\end{array}$ & ES & $\begin{array}{r}95 \% \text { confidence } \\
\text { interval }\end{array}$ & $\boldsymbol{P}_{\text {interaction }}$ \\
\hline All & & $9(154)$ & 0.81 & $0.32,1.3$ & \\
\hline \multirow[t]{2}{*}{ No. groups, timing } & 2-group posttest-only & $8(130)$ & 1.01 & $0.64,1.38$ & \\
\hline & 1-group pre-post & $1(24)$ & -0.05 & $-0.46,0.37$ & \\
\hline \multirow[t]{2}{*}{ Group allocation } & Yes & 7 (119) & 1.06 & $0.67,1.45$ & 0.41 \\
\hline & No & $1(11)$ & 0.53 & $-0.68,1.74$ & \\
\hline Participants & Postgraduate trainees & $9(154)$ & 0.81 & $0.32,1.3$ & \\
\hline \multirow[t]{2}{*}{ Cognitive interactivity } & High & $6(98)$ & 0.49 & $0.01,0.98$ & 0.02 \\
\hline & Low & $3(56)$ & 1.41 & $0.83,2$ & \\
\hline \multirow[t]{2}{*}{ Feedback } & High & $4(63)$ & 0.79 & $0.27,1.31$ & 0.85 \\
\hline & Low & $5(91)$ & 0.88 & $0.08,1.68$ & \\
\hline \multirow[t]{2}{*}{ Repetitions } & Many & $3(48)$ & 0.39 & $-0.36,1.13$ & 0.13 \\
\hline & Few & $6(106)$ & 1.05 & $0.62,1.48$ & \\
\hline Time learning & $\geq 5$ hours & $6(97)$ & 0.61 & $0.01,1.21$ & \\
\hline \multirow{2}{*}{ Assessment } & Blinded & $8(143)$ & 0.85 & $0.31,1.39$ & 0.63 \\
\hline & Not blinded & $1(11)$ & 0.53 & $-0.68,1.74$ & \\
\hline \multirow[t]{2}{*}{ NOS } & High $\geq 4$ & $5(93)$ & 1.2 & $0.76,1.65$ & 0.005 \\
\hline & Low $<4$ & $4(61)$ & 0.25 & $-0.24,0.74$ & \\
\hline \multirow[t]{2}{*}{ MERSQI } & High $\geq 12$ & $8(143)$ & 0.85 & $0.31,1.39$ & 0.63 \\
\hline & Low $<12$ & $1(11)$ & 0.53 & $-0.68,1.74$ & \\
\hline Effect size estimation & Precise & $8(133)$ & 0.69 & $0.21,1.16$ & \\
\hline
\end{tabular}

Table 2.4. Subgroup Analyses for Outcome: Process Behavior

\begin{tabular}{|c|c|c|c|c|c|}
\hline Feature & Subgroup & $\begin{array}{c}\text { No. studies (No. } \\
\text { participants) }\end{array}$ & ES & $\begin{array}{c}\text { 95\% confidence } \\
\text { interval }\end{array}$ & $\boldsymbol{P}_{\text {interaction }}$ \\
\hline All & & $14(533)$ & 0.94 & $0.6,1.28$ & \\
\hline \multirow[t]{3}{*}{ No. groups, timing } & 2-group posttest-only & $12(493)$ & 0.81 & $0.47,1.15$ & \\
\hline & 2-group pre-post & $1(16)$ & 2.25 & $1.24,3.26$ & \\
\hline & 1-group pre-post & $1(24)$ & 0.96 & $0.44,1.47$ & \\
\hline \multirow[t]{2}{*}{ Group allocation } & Randomized & $10(354)$ & 1.09 & $0.56,1.61$ & 0.13 \\
\hline & Nonrandomized & $3(155)$ & 0.59 & $0.24,0.95$ & \\
\hline Participants & Postgraduate trainees & $14(533)$ & 0.94 & $0.6,1.28$ & \\
\hline \multirow[t]{2}{*}{ Cognitive interactivity } & High & $9(431)$ & 0.95 & $0.53,1.37$ & 0.96 \\
\hline & Low & $5(102)$ & 0.93 & $0.26,1.6$ & \\
\hline \multirow[t]{2}{*}{ Feedback } & High & $6(293)$ & 1.14 & $0.45,1.83$ & 0.42 \\
\hline & Low & $8(240)$ & 0.82 & $0.46,1.18$ & \\
\hline \multirow[t]{2}{*}{ Repetitions } & Many $>10$ & $5(249)$ & 1.11 & $0.36,1.85$ & 0.56 \\
\hline & Few $\leq 9$ & $9(284)$ & 0.86 & $0.49,1.22$ & \\
\hline \multirow[t]{2}{*}{ Time learning } & $\geq 5$ hours & $6(101)$ & 1.42 & $0.98,1.86$ & 0.003 \\
\hline & $<5$ hours & $2(144)$ & 0.56 & $0.19,0.93$ & \\
\hline \multirow[t]{2}{*}{ Assessment } & Blinded & $8(135)$ & 1.4 & $0.99,1.81$ & 0.0005 \\
\hline & Not blinded & $6(398)$ & 0.5 & $0.21,0.79$ & \\
\hline \multirow[t]{2}{*}{ NOS } & High $\geq 4$ & $8(335)$ & 1.06 & $0.5,1.63$ & 0.41 \\
\hline & Low $<4$ & $6(198)$ & 0.78 & $0.38,1.17$ & \\
\hline \multirow[t]{2}{*}{ MERSQI } & High $\geq 12$ & 12 (419) & 1.01 & $0.6,1.42$ & 0.12 \\
\hline & Low $<12$ & $2(114)$ & 0.54 & $0.12,0.96$ & \\
\hline Effect size estimation & Precise & $11(472)$ & 0.82 & $0.45,1.2$ & \\
\hline
\end{tabular}

Copyright $@$ by the Association of American Medical Colleges. Unauthorized reproduction is prohibited. 
Supplemental digital content for Cook DA, Brydges R, Zendejas B, Hamstra SJ, Hatala R. Mastery learning for health professionals using technology-enhanced simulation: A systematic review and meta-analysis. Acad Med. 2013;88(8).

Table 2.5. Subgroup Analyses for Outcome: Patient Effects

\begin{tabular}{|c|c|c|c|c|c|}
\hline Feature & Subgroup & $\begin{array}{l}\text { No. studies (No. } \\
\text { participants) }\end{array}$ & ES & $\begin{array}{r}95 \% \text { confidence } \\
\text { interval }\end{array}$ & $\boldsymbol{P}_{\text {interaction }}$ \\
\hline All & & 11 (537) & 0.73 & $0.36,1.1$ & \\
\hline \multirow[t]{2}{*}{ No. groups, timing } & 2-group posttest-only & $8(491)$ & 0.69 & $0.3,1.08$ & \\
\hline & 1-group pre-post & $3(46)$ & 0.93 & $-0.34,2.2$ & \\
\hline \multirow[t]{2}{*}{ Group allocation } & Randomized & $4(244)$ & 0.39 & $0.13,0.64$ & 0.16 \\
\hline & Nonrandomized & $4(247)$ & 0.81 & $0.29,1.34$ & \\
\hline \multirow[t]{4}{*}{ Participants } & Postgraduate trainees & $9(515)$ & 0.81 & $0.41,1.2$ & \\
\hline & Practicing physicians & $1(5)$ & 1.18 & $-0.79,3.14$ & \\
\hline & Nurses & $1(17)$ & 0.09 & $-0.42,0.59$ & \\
\hline & Other & $1(17)$ & 0.09 & $-0.42,0.59$ & \\
\hline \multirow[t]{2}{*}{ Cognitive interactivity } & High & $9(486)$ & 0.88 & $0.47,1.29$ & 0.02 \\
\hline & Low & $2(51)$ & 0.16 & $-0.25,0.57$ & \\
\hline \multirow[t]{2}{*}{ Feedback } & High & $6(348)$ & 0.77 & $0.21,1.33$ & 0.88 \\
\hline & Low & $5(189)$ & 0.71 & $0.14,1.27$ & \\
\hline \multirow[t]{2}{*}{ Repetitions } & Many $>10$ & $5(250)$ & 0.73 & $0.13,1.33$ & 0.95 \\
\hline & Few $\leq 9$ & $6(287)$ & 0.76 & $0.31,1.21$ & \\
\hline \multirow[t]{2}{*}{ Time learning } & $\geq 5$ hours & $5(77)$ & 0.89 & $0.14,1.64$ & 0.93 \\
\hline & $<5$ hours & $4(241)$ & 0.85 & $0.3,1.4$ & \\
\hline \multirow[t]{2}{*}{ Assessment } & Blinded & $3(210)$ & 0.57 & $0.03,1.1$ & 0.60 \\
\hline & Not blinded & $8(327)$ & 0.76 & $0.3,1.21$ & \\
\hline \multirow[t]{2}{*}{ NOS } & High $\geq 4$ & $4(251)$ & 0.39 & $0.08,0.7$ & 0.11 \\
\hline & Low $<4$ & $7(286)$ & 0.85 & $0.37,1.32$ & \\
\hline \multirow[t]{2}{*}{ MERSQI } & High $\geq 12$ & $9(423)$ & 0.73 & $0.28,1.18$ & 0.78 \\
\hline & Low $<12$ & $2(114)$ & 0.82 & $0.39,1.24$ & \\
\hline Effect size estimation & Precise & $11(537)$ & 0.73 & $0.36,1.1$ & \\
\hline
\end{tabular}

* The studies summarized in all parts of Table 2 compared simulation-based mastery learning with no intervention.

Subgroup analyses grouped studies according to the presence or absence of the specified feature, and compared the mean effect size between these two groups. Analyses were performed separately for each outcome reported in $\geq 5$ studies. Instructional design subgroups (cognitive interactivity, feedback, repetitions, time learning) were determined by the presence of this feature in the mastery learning group. MERSQI = Medical Education Research Study Quality Instrument; NOS = modified Newcastle-Ottawa Scale.

Copyright $\odot$ by the Association of American Medical Colleges. Unauthorized reproduction is prohibited. 
Supplemental digital content for Cook DA, Brydges R, Zendejas B, Hamstra SJ, Hatala R. Mastery learning for health professionals using technology-enhanced simulation: A systematic review and meta-analysis. Acad Med. 2013;88(8).

\section{Supplemental Digital List 1}

References to Studies Included in the Systematic Review but Not Cited and Referenced in the Text of the Report *

55. Feil PH, Reed TR. The effectiveness of a leadup activity on performance in an operative preclinic laboratory course. J Dent Educ. 1989;53:565-568.

56. Ashurst N, Rout CC, Rocke DA, Gouws E. Use of a mechanical simulator for training in applying cricoid pressure. Br J Anaesth. 1996;77:468-472.

57. Ferlitsch A, Glauninger P, Gupper A, et al. Evaluation of a virtual endoscopy simulator for training in gastrointestinal endoscopy. Endoscopy. 2002;34:698-702.

58. Seymour NE, Gallagher AG, Roman SA, et al. Virtual reality training improves operating room performance: results of a randomized, double-blinded study. Ann Surg. 2002;236:458-463.

59. Neumann M, Siebert T, Rausch J, et al. Scorecard endoscopy: a pilot study to assess basic skills in trainees for upper gastrointestinal endoscopy. Langenbeck's Archives of Surgery. 2003;387:386-391.

60. Jacomides L, Ogan K, Cadeddu JA, Pearle MS. Use of a virtual reality simulator for ureteroscopy training. J Urol. 2004;171:320-323.

61. Mayo PH, Hackney JE, Mueck JT, Ribaudo V, Schneider RF. Achieving house staff competence in emergency airway management: Results of a teaching program using a computerized patient simulator. Crit Care Med. 2004;32:2422-2427.

62. Ahlberg G, Hultcrantz R, Jaramillo E, Lindblom A, Arvidsson D. Virtual reality colonoscopy simulation: a compulsory practice for the future colonoscopist? Endoscopy. 2005;37:1198-1204.

63. Korndorffer JR, Jr., Hayes DJ, Dunne JB, Sierra R, Touchard CL, Markert RJ, et al. Development and transferability of a cost-effective laparoscopic camera navigation simulator. Surg Endosc. 2005;19:161-167.

64. Korndorffer JR, Jr., Dunne JB, Sierra R, Stefanidis D, Touchard CL, Scott DJ. Simulator training for laparoscopic suturing using performance goals translates to the operating room. J Am Coll Surg. 2005;201:23-29.

65. Stefanidis D, Korndorffer JR, Jr., Sierra R, Touchard C, Dunne JB, Scott DJ. Skill retention following proficiencybased laparoscopic simulator training. Surgery. 2005;138:165-170.

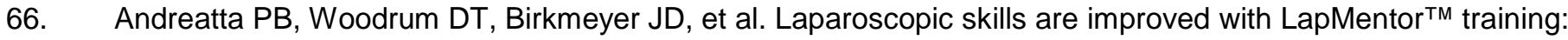
results of a randomized, double-blinded study. Ann Surg. 2006;243:854-860.

Copyright $\odot$ by the Association of American Medical Colleges. Unauthorized reproduction is prohibited. 
Supplemental digital content for Cook DA, Brydges R, Zendejas B, Hamstra SJ, Hatala R. Mastery learning for health professionals using technology-enhanced simulation: A systematic review and meta-analysis. Acad Med. 2013;88(8).

67. Stefanidis D, Sierra R, Korndorffer JR, Jr., et al. Intensive continuing medical education course training on simulators results in proficiency for laparoscopic suturing. Am J Surg. 2006;191:23-27.

68. Stefanidis D, Korndorffer JR, Jr., Black FW, et al. Psychomotor testing predicts rate of skill acquisition for proficiency-based laparoscopic skills training. Surgery. 2006;140:252-262.

69. Wayne DB, Butter J, Siddall VJ, et al. Mastery learning of advanced cardiac life support skills by internal medicine residents using simulation technology and deliberate practice. J Gen Intern Med. 2006;21:251-256.

70. Aggarwal R, Ward J, Balasundaram I, Sains P, Athanasiou T, Darzi A. Proving the effectiveness of virtual reality simulation for training in laparoscopic surgery. Ann Surg. 2007;246:771-779.

71. Ahlberg G, Enochsson L, Gallagher AG, et al. Proficiency-based virtual reality training significantly reduces the error rate for residents during their first 10 laparoscopic cholecystectomies. Am J Surg. 2007;193:797-804.

72. Cosman PH, Hugh TJ, Shearer CJ, Merrett ND, Biankin AV, Cartmill JA. Skills acquired on virtual reality laparoscopic simulators transfer into the operating room in a blinded, randomised, controlled trial. Stud Health Technol Inform. 2007;125:76-81.

73. Ganai S, Donroe JA, St. Louis MR, Lewis GM, Seymour NE. Virtual-reality training improves angled telescope skills in novice laparoscopists. Am J Surg. 2007;193:260-265.

74. Kory PD, Eisen LA, Adachi M, Ribaudo VA, Rosenthal ME, Mayo PH. Initial airway management skills of senior residents: simulation training compared with traditional training. Chest. 2007;132:1927-1931.

75. Scott DJ, Goova MT, Tesfay ST. A cost-effective proficiency-based knot-tying and suturing curriculum for residency programs. J Surg Res. 2007;141:7-15.

76. Yi SY, Ryu KH, Woo HS, Ahn W, Kim WS, Lee DY. Quantitative analysis of colonoscopy skills using the KAISTEwha Colonoscopy Simulator II. Paper presented at: Proceedings of the Frontiers in the Convergence of Bioscience and Information Technologies (FBIT), 2007.

77. Goova MT, Hollett LA, Tesfay ST, et al. Implementation, construct validity, and benefit of a proficiency-based knot-tying and suturing curriculum. J Surg Educ. 2008;65:309-315.

78. Scott DJ, Ritter EM, Tesfay ST, Pimentel EA, Nagji A, Fried GM. Certification pass rate of 100\% for fundamentals of laparoscopic surgery skills after proficiency-based training. Surg Endosc. 2008;22:1887-1893.

79. Smith KK, Gilcreast D, Pierce K. Evaluation of staff's retention of ACLS and BLS skills. Resuscitation. 2008;78:5965.

Copyright $\odot$ by the Association of American Medical Colleges. Unauthorized reproduction is prohibited. 
Supplemental digital content for Cook DA, Brydges R, Zendejas B, Hamstra SJ, Hatala R. Mastery learning for health professionals using technology-enhanced simulation: A systematic review and meta-analysis. Acad Med. 2013;88(8).

80. Stefanidis D, Acker C, Heniford TB. Proficiency-based laparoscopic simulator training leads to improved operating room skill that is resistant to decay. Surgical Innovation. 2008;15:69-73.

81. Van Sickle KR, Ritter EM, Baghai M, et al. Prospective, randomized, double-blind trial of curriculum-based training for intracorporeal suturing and knot tying. J Am Coll Surg. 2008;207:560-568.

82. Wayne DB, Barsuk JH, O'Leary KJ, Fudala MJ, McGaghie WC. Mastery learning of thoracentesis skills by internal medicine residents using simulation technology and deliberate practice. J Hosp Med. 2008;3:48-54.

83. Yi SY, Ryu KH, Na YJ, et al. Improvement of colonoscopy skills through simulation-based training. Stud Health Technol Inform. 2008;132:565-567.

84. Barsuk JH, Ahya SN, Cohen ER, McGaghie WC, Wayne DB. Mastery learning of temporary hemodialysis catheter insertion by nephrology fellows using simulation technology and deliberate practice. Am J Kidney Dis. 2009;54:70-76.

85. Barsuk JH, McGaghie WC, Cohen ER, O'Leary KJ, Wayne DB. Simulation-based mastery learning reduces complications during central venous catheter insertion in a medical intensive care unit. Crit Care Med. 2009;37:2697-2701.

86. Barsuk JH, Cohen ER, Feinglass J, McGaghie WC, Wayne DB. Use of simulation-based education to reduce catheter-related bloodstream infections. Arch Intern Med. 2009;169:1420-1423.

87. Barsuk JH, McGaghie WC, Cohen ER, Balachandran JS, Wayne DB. Use of simulation-based mastery learning to improve the quality of central venous catheter placement in a medical intensive care unit. J Hosp Med. 2009;4:397-403.

88. Britt RC, Novosel TJ, Britt LD, Sullivan M. The impact of central line simulation before the ICU experience. Am J Surg. 2009;197:533-536.

89. Castellvi AO, Hollett LA, Minhajuddin A, Hogg DC, Tesfay ST, Scott DJ. Maintaining proficiency after fundamentals of laparoscopic surgery training: a 1-year analysis of skill retention for surgery residents. Surgery. 2009;146:387-393.

90. Duncan DR, Morgenthaler TI, Ryu JH, Daniels CE. Reducing iatrogenic risk in thoracentesis: establishing best practice via experiential training in a zero-risk environment. Chest. 2009;135:1315-1320.

91. Essani R, Scriven RJ, McLarty AJ, Merriam LT, Ahn H, Bergamaschi R. Simulated laparoscopic sigmoidectomy training: responsiveness of surgery residents. Dis Colon Rectum. 2009;52:1956-1961.

Copyright $\odot$ by the Association of American Medical Colleges. Unauthorized reproduction is prohibited. 
Supplemental digital content for Cook DA, Brydges R, Zendejas B, Hamstra SJ, Hatala R. Mastery learning for health professionals using technology-enhanced simulation: A systematic review and meta-analysis. Acad Med. 2013;88(8).

92. Feudner EM, Engel C, Neuhann IM, Petermeier K, Bartz-Schmidt K-U, Szurman P. Virtual reality training improves wet-lab performance of capsulorhexis: results of a randomized, controlled study. Graefes Arch Clin Exp Ophthalmol. 2009;247:955-963.

93. Hogle NJ, Chang L, Strong VEM, et al. Validation of laparoscopic surgical skills training outside the operating room: a long road. Surg Endosc. 2009;23:1476-1482.

94. Larsen CR, Soerensen JL, Grantcharov TP, et al. Effect of virtual reality training on laparoscopic surgery: randomised controlled trial BMJ (Clinical research ed). 2009;338:b1802.

95. Lubin J, Carter R. The feasibility of daily mannequin practice to improve intubation success. Air Med J. 2009;28:195-197.

96. Naylor RA, Hollett LA, Valentine RJ, et al. Can medical students achieve skills proficiency through simulation training? Am J Surg. 2009;198:277-282.

97. Pauly-O'Neill S. Beyond the five rights: improving patient safety in pediatric medication administration through simulation. Clinical Simulation in Nursing. 2009;5:e181-e186.

98. Stefanidis D, Scott DJ, Korndorffer JR, Jr. Do metrics matter? Time versus motion tracking for performance assessment of proficiency-based laparoscopic skills training. Simul Healthc. 2009;4:104-108.

99. Yi SY, Ryu KH, Woo HS, et al. Sectional analysis of learning on the KAIST-Ewha Colonoscopy Simulation II. Stud Health Technol Inform. 2009;142:432-434.

100. Butter J, McGaghie WC, Cohen ER, Kaye ME, Wayne DB. Simulation-based mastery learning improves cardiac auscultation skills in medical students. J Gen Intern Med. 2010;25:780-785.

101. Evans LV, Dodge KL, Shah TD, et al. Simulation training in central venous catheter insertion: improved performance in clinical practice. Acad Med. 2010;85:1462-1469.

102. Fried MP, Sadoughi B, Gibber MJ, et al. From virtual reality to the operating room: the endoscopic sinus surgery simulator experiment. Otolaryngol Head Neck Surg. 2010;142:202-207.

103. Kallstrom R, Hjertberg H, Svanvik J. Impact of virtual reality-simulated training on urology residents' performance of transurethral resection of the prostate. J Endourol. 2010;24:1521-1528.

104. Rosenthal ME, Ritter EM, Goova MT, Castellvi AO, Tesfay ST, Pimentel EA, et al. Proficiency-based Fundamentals of Laparoscopic Surgery skills training results in durable performance improvement and a uniform certification pass rate. Surg Endosc. 2010;24:2453-2457.

Copyright $\odot$ by the Association of American Medical Colleges. Unauthorized reproduction is prohibited. 
Supplemental digital content for Cook DA, Brydges R, Zendejas B, Hamstra SJ, Hatala R. Mastery learning for health professionals using technology-enhanced simulation: A systematic review and meta-analysis. Acad Med. 2013;88(8).

105. Sroka G, Feldman LS, Vassiliou MC, Kaneva PA, Fayez R, Fried GM. Fundamentals of laparoscopic surgery simulator training to proficiency improves laparoscopic performance in the operating room-a randomized controlled trial. Am J Surg. 2010;199:115-120.

106. Stefanidis D, Acker CE, Greene FL. Performance goals on simulators boost resident motivation and skills laboratory attendance. J Surg Educ. 2010;67:66-70.

107. Unalan PC, Akan K, Orhun H, et al. A basic arthroscopy course based on motor skill training. Knee Surg Sports Traumatol Arthrosc. 2010;18:1395-1399.

108. Vankipuram M, Kahol K, McLaren A, Panchanathan S. A virtual reality simulator for orthopedic basic skills: A design and validation study. Journal of Biomedical Informatics. 2010;43:661-668.

109. Maschuw K, Schlosser K, Kupietz E, Slater EP, Weyers P, Hassan I. Do Soft Skills Predict Surgical Performance? A Single-Center Randomized Controlled Trial Evaluating Predictors of Skill Acquisition in Virtual Reality Laparoscopy. World J Surg. 2011;35:480-486.

110. Panait L, Hogle NJ, Fowler DL, Bell RL, Roberts KE, Duffy AJ. Completion of a novel, virtual-reality-based, advanced laparoscopic curriculum improves advanced laparoscopic skills in senior residents. J Surg Educ. 2011;68:121-125.

* Reference 1-54 are listed in the text of the report. For key features of the 82 studies featured in this systematic review, see Supplemental Digital Table 1. For a general description of these same studies, see Table 1 in the report.

Copyright $\odot$ by the Association of American Medical Colleges. Unauthorized reproduction is prohibited. 